\title{
Modeling the discontinuous individual channel injection into fin-and-tube evaporators for residential air-conditioning
}

\author{
Martin Ryhl Kærn ${ }^{\dagger} \ddagger \quad$ Brian Elmegaard ${ }^{\dagger}$ \\ ${ }^{\dagger}$ Technical University of Denmark, Department of Mechanical Engineering \\ Nils Koppels Allé Bygn. 403, DK-2800 Lyngby, Denmark, e-mail: pmak@mek .dtu.dk \\ ${ }^{\ddagger}$ Former address: Danfoss A/S, Refrigeration and Air-Conditioning \\ Nordborgvej 81, DK-6430 Nordborg, Denmark
}

\begin{abstract}
In this paper a working principle based upon the novel expansion and distributor device EcoFlow ${ }^{\mathrm{TM}}$ is analyzed. The device enables compensation of flow maldistribution by control of individual channel superheat. The working principle is discontinuous liquid injection (pulsating flow) into each individual channels during a specified cycle time. Moreover, the influence of the injection cycle time is investigated together with an optional secondary flow into the other channels with regards to cooling capacity, overall UA-value and COP.

The results showed spurious fluctuations in pressure when simulating the pulsating flow, thus the dynamic behavior in the mixture two-phase flow model is insufficient to model the discontinuous liquid injection principle. Despite, the fluctuations and imperfections of the model we found that the cycle time should be kept as low as possible and that the optional secondary flow increases performance. Moreover, the paper reports on the applicability of Modelica developed models to analyze and optimize the working principle and design of expansion devices such that Modelica may be used in future development of novel discontinuous expansion devices.
\end{abstract}

Keywords: refrigeration; air-conditioning; evaporator; two-phase flow; liquid injection; pulsation; transient; dynamic; modeling; simulation; Modelica.

\section{Nomenclature}

\section{Roman}

A cross-sectional area $\left(\mathrm{m}^{2}\right)$

$c_{p} \quad$ specific heat capacity $\left(\mathrm{J} \mathrm{kg}^{-1} \mathrm{~K}^{-1}\right)$

$C$ capacitance flow $\left(\mathrm{W} \mathrm{K}^{-1}\right)$
COP coefficient of performance (-)

$D \quad$ inner tube outer diameter (m)

$d \quad$ inner tube inner diameter $(\mathrm{m})$

$F_{w} \quad$ wall friction force $\left(\mathrm{N} \mathrm{m}^{-3}\right)$

$F_{o} \quad$ orifice flow ratio parameter (-)

$g \quad$ gravitational acceleration $\left(\mathrm{m} \mathrm{s}^{-2}\right)$

$\dot{H} \quad$ enthalpy flow (W)

$h \quad$ specific mixed-cup enthalpy $\left(\mathrm{J} \mathrm{kg}^{-1}\right)$

$\bar{h} \quad$ specific in situ mixture enthalpy $\left(\mathrm{J} \mathrm{kg}^{-1}\right)$

$h_{\mathrm{tc}} \quad$ heat transfer coefficient $\left(\mathrm{W} \mathrm{m}^{-2} \mathrm{~K}^{-1}\right)$

I momentum flow $(\mathrm{N})$

$K \quad$ orifice flow coefficient

$k \quad$ thermal conductivity ( $\mathrm{W} \mathrm{m}^{-1} \mathrm{~K}^{-1}$ )

$M \quad$ mass $(\mathrm{kg})$

$\dot{m} \quad$ mass flow rate $\left(\mathrm{kg} \mathrm{s}^{-1}\right)$

NTU number of transfer units (-)

OD opening degree (\%)

$P \quad$ channel perimeter $(\mathrm{m})$

$p \quad$ pressure $(\mathrm{Pa})$

$\dot{Q} \quad$ heat flow rate $(\mathrm{W})$

$q_{w}^{\prime \prime} \quad$ wall heat flux $\left(\mathrm{W} \mathrm{m}^{-2}\right)$

$R \quad$ thermal resistance $\left(\mathrm{K} \mathrm{W}^{-1}\right)$

$S \quad$ slip ratio (-)

$T$ temperature (K)

$t \quad$ time (s)

$U \quad$ velocity $\left(\mathrm{m} \mathrm{s}^{-1}\right)$

UA overall UA-value $\left(\mathrm{W} \mathrm{K}^{-1}\right)$

$x \quad$ vapor quality (-)

$z \quad$ axial channel length (m)

Greek

$\alpha \quad$ void fraction (-)

$\varepsilon \quad$ effectiveness (-)

$\Theta \quad$ distribution vector (-)

$\rho \quad$ density $\left(\mathrm{kg} \mathrm{m}^{-3}\right)$

$\bar{\rho} \quad$ mixture density $\left(\mathrm{kg} \mathrm{m}^{-3}\right)$ 
$\rho^{\prime} \quad$ momentum density $\left(\mathrm{kg} \mathrm{m}^{-3}\right)$

$\theta \quad$ angle to horizontal plane (deg.)

\begin{tabular}{ll}
\multicolumn{2}{l}{ Subscripts } \\
ax & axial \\
$c$ & condensation \\
cyc & cycle \\
$e$ & evaporation \\
exp & experiment \\
$f$ & saturated liquid \\
$g$ & saturated gas \\
$H$ & homogeneous \\
damp & dampening \\
inj & injection \\
rad & radial \\
ss & steady state \\
tot & total \\
$w$ & wall
\end{tabular}

\section{Introduction}

Flow maldistribution in fin-and-tube evaporators has been shown by many investigators to reduce the performance of air-conditioning systems in terms of cooling capacity and COP. Furthermore, compensation of flow maldistribution by control of individual channel superheat has been shown to recover the penalties of flow maldistribution significantly $[1,2,3]$. Perfect control of individual channel superheats means that a thermostatic or electronic expansion valve is located on each evaporator channel and thus controls each superheat to be the same. It is not beneficial for economic reasons to install an expansion valve for each channel. Therefore, the discontinuous liquid injection principle is studied in this paper as a promising method for compensation by control of individual channel superheat. On the other hand, the tube circuitry of fin-and-tube evaporators may be optimized to compensate flow maldistribution by design [4] such that equal channel superheats occur, however, it does not ensure equal channel superheats at part-load or offdesign conditions.

The focus of the current study is to gain more understanding and insight in the discontinuous liquid injection into each evaporator channels and its implications for evaporator design and system performance in terms of overall UA-value, cooling capacity and COP. We will investigate implications for two standard tube circuitries namely the face split and the interlaced evaporator, see figure 1. Especially, we strive to optimize the discontinuous liquid injection principle by study- ing the effects of different specifications (cycle time and optional secondary flow) and provide guidelines for optimal energy efficiency. For simplicity we do not consider actual flow maldistribution when evaluating the effect of cycle time and optional secondary flow. The injection principle is essentially two-phase flow pulsations and the study may show the potential of increasing capacity and COP by employing pulsations to the flow.

The modeling of the liquid injection dynamics showed spurious fluctuations in pressure, which have not been observed as high in any similar experiments carried out at Danfoss. The current analysis should therefore be seen as a first study of the injection dynamics with the current model approach and limitations. When simulating the injection dynamics, we must keep in mind that the correlations for heat transfer, friction and void may become invalid at large transients in mass flow, since they are developed from steady state experiments. Furthermore, the discontinuous refrigerant injection is essentially pulsating twophase flow, and the significance of the liquid/vapor interfacial dynamics may become important such as interfacial friction and drag and/or thermodynamic nonequilibrium effects. These phenomenons are not included in the typical mixture two-phase flow model used in many Modelica libraries, and also used in the current study (developed in Kærn [3]).

\subsection{Liquid injection principle}

The liquid injection principle is based on the recently developed Danfoss product $\left(\right.$ EcoFlow $\left.^{\mathrm{TM}}[5]\right)$. Actually, the EcoFlow valve does not measure the individual channel superheats but only the overall superheat. Furthermore, it does not provide continuous refrigerant flow in each channel, but rather discontinuous individual channel injection (modulation of each channel flow) with optional secondary flow to the other channels. The optimal distribution of mass flow rate (at flow maldistribution) is then found from a distribution analysis performed at specific time intervals during operation, see Mader and Thybo [6]. The distribution analysis is essentially carried out by control algorithms, where the importance of each individual channel on the overall superheat is measured in order to find the optimal distribution. The individual channel superheats become the same at the optimal mass flow distribution.

The individual injection is performed by a stepper motor (48 steps per revolution), which rotates the distributor disc, see figure 2a. The EcoFlow valve comes 

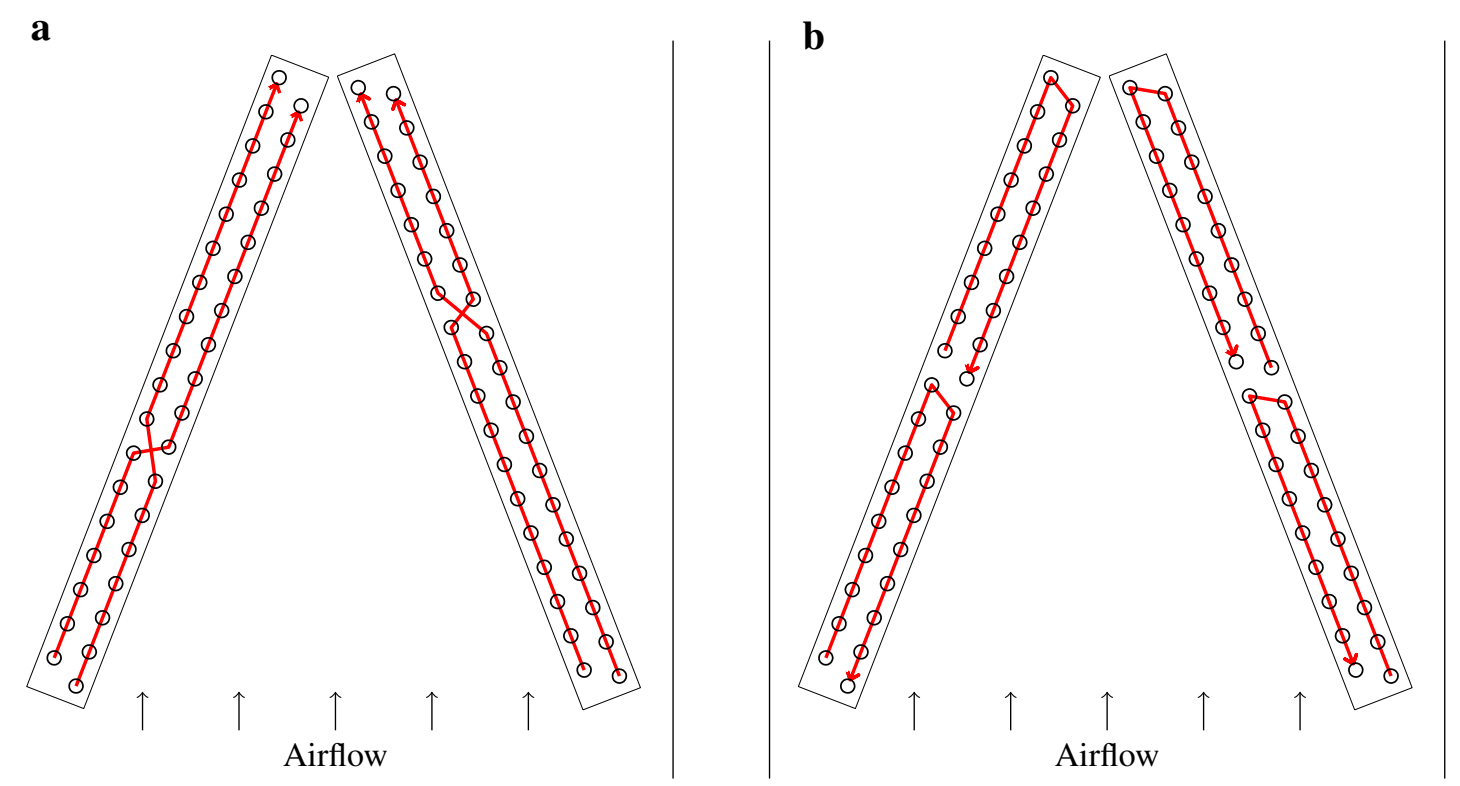

Figure 1: Tube circuitries of the interlaced evaporator (a) and the face split evaporator (b).

in two different designs, i.e. a multi-orifice (MO) design (main orifice + secondary orifices) and a singleorifice (SO) design (main orifice only), see figure $2 b$ and 2c. The orifice size of the SO design is larger, since more refrigerant needs to pass through the main orifice. The SO design enables the possibility of individual channel defrost during cooling operation (no defrost periods) for the face split evaporator only, see figure $1 \mathrm{~b}$. As we shall see later, the results show that the performance in steady state without considering frost build-up becomes a bit smaller when using the SO concept. Furthermore, all orifices of both designs are closed in between each channel injection.

\subsection{Objectives and content}

The first objective is to evaluate the effect of the cycle time for the MO and SO design concepts, i.e. the time it takes for one revolution. The second objective is to evaluate the size of the secondary orifices in the MO design compared to the main orifice. The questions that are sought to be answered are:

- What is the minimum cycle time for discontinuous liquid injection? Too large cycle times will cause too much dry-out of the channels.

- Does capacity decrease or increase by the discontinuous liquid injection (pulsating flow)?

- How much refrigerant should pass through the main and secondary orifices in the MO design?

Note that the results is focused on the steady state performance in terms of overall UA-value, cooling ca- pacity and COP, where the dynamics of the refrigerant injection is modeled.

The paper starts by a brief description of the liquid injection modeling and use of experimental results for evaluating orifice flow coefficients for the actual MO and SO designs. Then the pressure fluctuations caused by the liquid injection modeling is considered and compared to experiments using an earlier MO design and performed on the interlaced tube circuitry. Finally, the effect of the cycle time and flow ratio between main and secondary orifices of the MO concept are investigated.

\section{Modeling approach}

This section describes the model that was implemented in the Modelica language of the discontinuous liquid injection principle. Furthermore, the system model is described with focus on the evaporator.

\subsection{Injection modeling}

This section describes the experimental data reduction that was performed of actual EcoFlow capacity tests, in order to obtain the orifice flow coefficients for both MO and SO designs (see figure 2). The goal of the data reduction is to compute the mass flow through the main orifice and secondary orifices at different pressure levels and opening degrees (when the expansion valve is open only). The capacity tests provide continuous capacity (evaporation of refrigerant) or mass 
$\mathbf{a}$

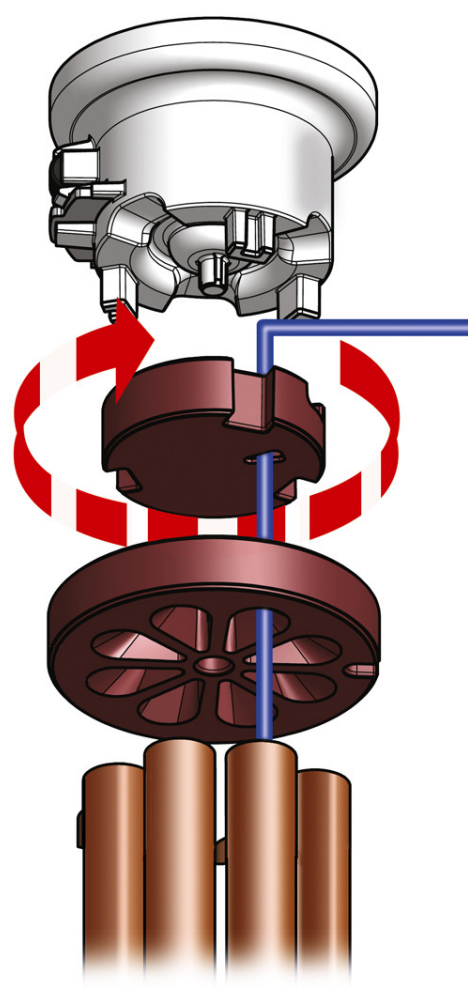

b

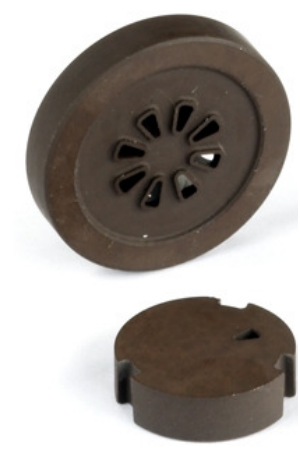

c

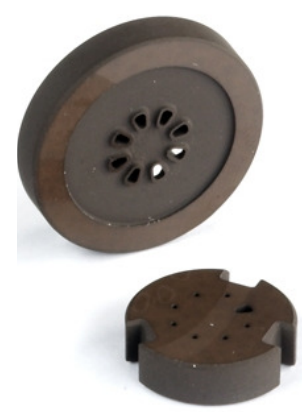

Figure 2: EcoFlow distribution method and refrigerant flow through discs (a), single-orifice (SO) discs (b) and multi-orifice (MO) discs (c).

flow rate through the valve, but we are only interested in the mass flow through the valve when it is open. When knowing the orifice flow coefficient $K$, the mass flow through the valve may be computed by the single phase orifice equation as

$$
\dot{m}_{\text {open }}=K A \sqrt{2 \rho_{f}\left(p_{\text {in }}-p_{\text {out }}\right)}
$$

where $A$ is the flow area of the orifice, $\rho_{f}$ is the saturated liquid density, $p_{\text {in }}$ and $p_{\text {out }}$ are the pressure at inlet and outlet of the valve. The use of equation 1 is the standard method of developing empirical equations to predict mass flow rate through orifices [7] even in refrigerant expansion devices $[8,9]$.

Two-phase flow effects such as partial vaporization (flashing) are included in the flow coefficient. Furthermore, the capacity tests of the orifice discs were only carried out at standard conditions. It means that $K$ will not be dependent on the pressure levels, and is thus assumed to be constant at different pressure levels. The standard conditions for these capacity tests are: Evaporation at $5^{\circ} \mathrm{C}$, condensation at $32^{\circ} \mathrm{C}, 4 \mathrm{~K}$ subcooling and no superheat. The relation between the experimental mass flow rate and valve capacity is thus

$$
\dot{Q}_{\text {exp }}=\dot{m}_{\text {exp }}\left[h_{g}\left(p_{\text {out }}\right)-h\left(p_{\text {in }}, T_{\text {in }}\right)\right]
$$

The stepper motor has 48 steps per revolution equaling 7.5 degree rotation per step. The step time is 10 $\mathrm{ms}$ per step, i.e. a minimum of $480 \mathrm{~ms}$ per revolution (minimum cycle time). Due to the opening and closing of the valve, the liquid refrigerant before the valve will create a fluid hammer (also called a hydraulic shock). The moving liquid is suddenly forced to stop, and the pressure builds up before the valve and a pressure wave will propagate upstream. In order to eliminate the peak forces acting on the valve, the speed of the stepper motor is dampened as the valve opens and closes.

To find the actual mass flow through the valve when open we need to know the opening time of the valve (injection time). The actual injection time is a function of cycle time, opening degree, damping time and step time of the stepper motor. A detailed description is given in Kærn [3], however, it is simply a matter of tracking the time when open and closed. When the injection time is known the mass flow through the valve when open may be computed by mass continuity as

$$
\dot{m}_{\text {open }}=\dot{m}_{\text {exp }} \frac{t_{\text {cyc }}}{t_{\text {inj }}}
$$

and used in equation 1 to compute the flow coefficient $K$ for the total flow through main and secondary orifices. The flow coefficient is thus for actual design and number of discharge channels (EcoFlow is made with up to 8 discharge channels), and is a function of opening degree, cycle time, step time and damping time.

In this paper we only consider four channel evaporators, i.e. two coils with two channels each. Therefore, the flow coefficients were only computed on the four channel orifice discs with MO and SO designs. The standard EcoFlow time settings are a step time of 10 $\mathrm{ms}$ and a damping time of $120 \mathrm{~ms}$ for both opening and closing. Using the capacity tests, we computed the flow coefficients for the total flow as function of opening degree for cycle times 6, 10 and 20 seconds for both $\mathrm{MO}$ and SO designs. For the SO design the total flow comes through the main orifice, however, for the MO design we need additional information on how much flow that goes into the main and secondary orifices, respectively.

Fortunately, a capacity test was also performed at steady state conditions, i.e. no rotation of the distributor disc and fully open continuous flow. The test was done at all orifices open, but also at main orifice closed, which gives us the flow ratio parameter be- 
tween the main orifice flow and total flow in steady state as

$$
F_{o}=\frac{\dot{m}_{\text {main }, \mathrm{ss}}}{\dot{m}_{\mathrm{tot}, \mathrm{ss}}}=0.492
$$

The ratio is assumed to be independent of the cycle time and damping time, and thus directly used to distribute the total mass flow to the main and the secondary orifices when the valve is open. The total mass flow when the valve is open and the corresponding steady state mass flow are shown on figure 3a. Figure $3 b$ shows the corresponding flow coefficients.

$\mathbf{a}$

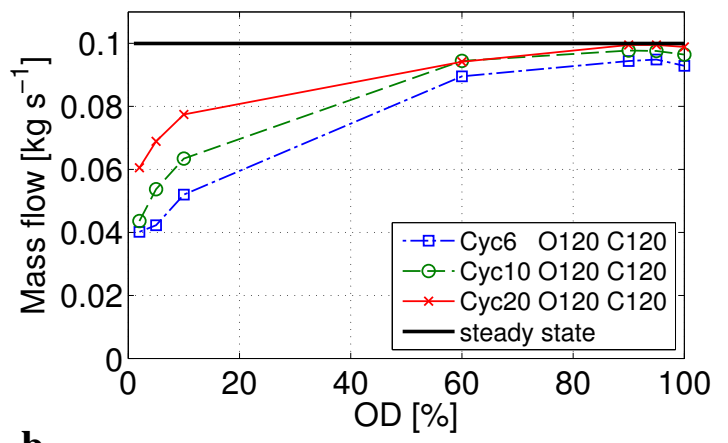

b

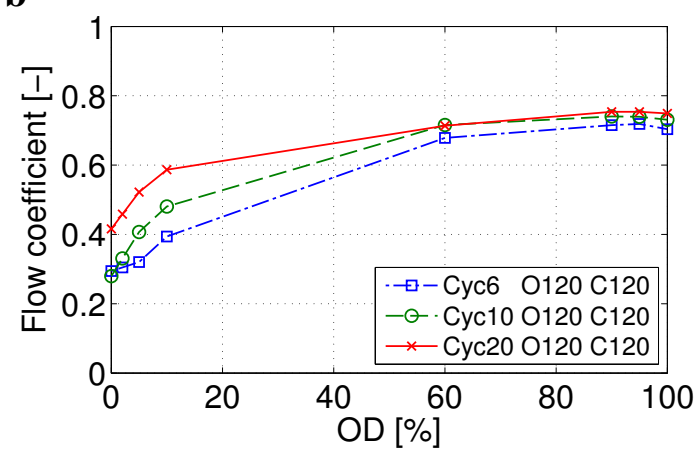

Figure 3: Total mass flow rate when valve is open (a) and flow coefficients (b) as function of opening degree (MO design); $\mathrm{Cyc}=$ cycle time [s], $\mathrm{O}=$ opening damping time $[\mathrm{ms}], \mathrm{C}=$ closing damping time $[\mathrm{ms}]$.

We assume that the accelerational effects of the fluid at opening and closing may cause the differences in the flow coefficients and mass flows, which tends to differ more at low opening degree, where the accelerational effects should play a larger role compared to the actual mass transferred through the valve. As expected, the mass flow curves are below the steady state mass flow and becomes closer at high opening degree, where the opening and closing have smaller influence. Unfortunately, there were no measurements between $10 \%$ and $60 \%$ opening degree.

The expansion process may experience choking of the flow, i.e. the mass flow may not increase by decreasing the downstream pressure and is only a function of upstream conditions. Using the above modeling approach does not include the choking phenomenon and the mass flow is essentially a function of pressure difference and flow coefficient. It is thus assumed that choking of the flow is not existing.

\subsubsection{Implementation}

The implementation of the liquid injection model in Modelica is done by using the CombiTable1D model from the Standard Modelica Library, i.e. onedimensional linear table interpolation of the flow coefficients. The mass flow rates through the main orifice and secondary orifices (MO) are then computed using equation 1 and 4 . Now it is just a matter of computing the individual channel opening and closing time during each cycle. A distribution vector is defined as

$$
\sum_{i=1}^{N} \Theta_{i}=1
$$

which determines the time period associated with each channel $t_{\text {tube }, i}$ as

$$
t_{\text {tube }, i}=t_{\text {cyc }} \Theta_{i}
$$

where $i$ denotes the channel number and $N$ the total number of channels. The injection time for each channel is computed by

$$
\begin{aligned}
t_{\text {inj }, i}= & \left(t_{\text {tube }, i}-\frac{N_{\text {step }} t_{\text {step }}}{N}-\frac{N_{\text {damp }} t_{\text {damp }}}{N}\right) \frac{\mathrm{OD}}{100} \\
& +\frac{N_{\text {damp }} t_{\text {damp }}}{N}
\end{aligned}
$$

The first term in the parenthesis is the controllable time per channel (minimum cycle time subtracted) times opening degree. The second term counts for the additional mass flow that would occur even though the opening degree is zero. The dampening time occurs from approximately $70 \%$ to $100 \%$ opening area of the orifice (as the disc turn). For simplicity, the additional mass flow is assumed to be the mass flow when fully open times the damping time.

The opening of each channel is assumed to occur at $t_{\text {tube }, i} / 2-t_{\text {inj, }, i} / 2$. The closing is then at $t_{\text {tube }, i} / 2+$ $t_{\mathrm{inj}, i} / 2$. The changes in mass flow rate are made smooth by use of the first order continuous functions as described in $[3,10]$ for numerical reasons. The transition time was chosen to be 0.1 seconds.

Figure 4 shows some examples of the MO liquid injection model at a cycle time of 10 seconds. It illustrates the working principle of the liquid injection 
a

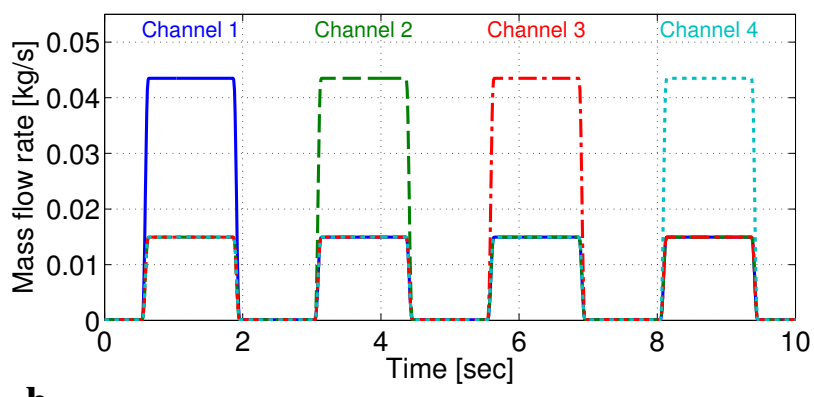

b

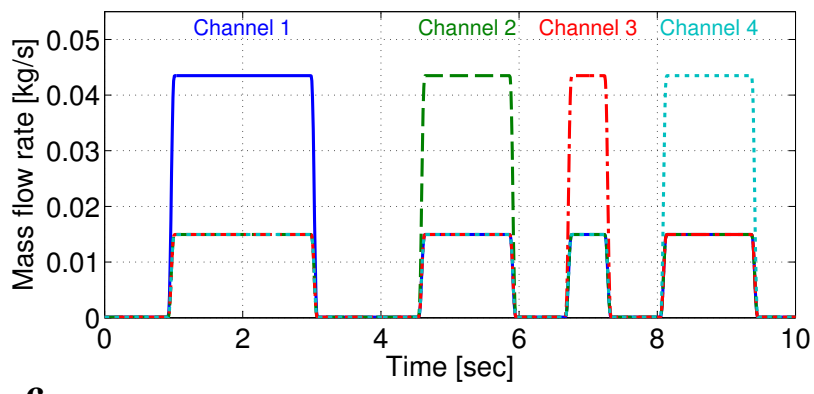

c

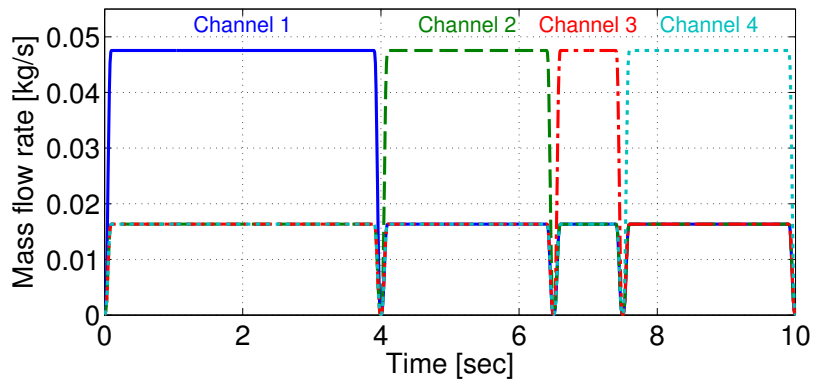

Figure 4: Mass flow distributions for liquid injection model with $\mathrm{MO}$ design at cycletime $=$ $10 \mathrm{~s} ; \Theta=[0.25,0.25,0.25,0.25], \mathrm{OD}=50 \% \quad$ (a); $\Theta=[0.4,0.25,0.1,0.25], \mathrm{OD}=50 \% \quad(\mathrm{~b}) ; \quad \Theta=$ $[0.4,0.25,0.1,0.25], \mathrm{OD}=100 \%(\mathrm{c}) ; p_{e}=9.3 \mathrm{bar}$ and $p_{c}=19.8$ bar (standard condition).

model as the opening degree and the distribution vector are changed. Throughout this paper we do not consider compensation of flow maldistribution, thus the liquid injection model runs in even flow mode (figure 4a) and the distribution vector becomes $\Theta=$ $[0.25,0.25,0.25,0.25]$. In compensating flow mode the values in the distribution vector need be controlled in the numerical model according to the individual channel superheat.

\subsection{Model setup}

The numerical model is described in Kærn et al. [11] for a co-axial evaporator and has been updated as described in Kærn [3] to model the full system (condenser and compressor also) and the tube circuitries of the interlaced and face split evaporators, see figure 1. The model is implemented in the Modelica language and Dymola 7.4 [12] is used as simulator. The Modelica language facilitates object-oriented programming, which is important for model reuse and extension. Dymola has been well tested within the field of air-conditioning and refrigeration [13, 10]. Thermophysical properties for R410A are obtained from the Refeqns package [14]. In order to model the refrigerant distribution and circuitry in the evaporator a dynamic distributed one-dimensional mixture finite volume model was chosen. For the condenser, the simpler moving boundary model of Zhang and Zhang [15] was chosen, which averages the vapor, two-phase and liquid regions. The models of the expansion and compressor are quasi-static. Momentum transfer and frictional pressure drop are only addressed in the evaporator tubes, U-bends and feeder tubes, in order to predict the mass flow distribution in the evaporator. Furthermore, the void fraction model by Zivi (1964) is used to model the refrigerant charge of both condenser and evaporator.

Since the evaporator pressure showed spurious fluctuations when simulating the injection principle, we included the refrigerant flow equations and implementation for the evaporator model in the appendix such that these may be studied by the reader. Furthermore, we did not use the Modelica stream prefix. Since the compressor runs at constant speed, we did not observe flow reversal during the flow pulsations.

\subsubsection{Geometry and correlations}

Table 1 shows the main geometry of the test case evaporator and condenser. The tube inner walls are smooth. Furthermore, the feeder tubes to the evaporator have an internal diameter of $3 \mathrm{~mm}$ and a length of $300 \mathrm{~mm}$. The manifold inner and outer diameter is $16 \mathrm{~mm}$ and $19 \mathrm{~mm}$, respectively, and its length is $5 \mathrm{~m}$ from the evaporator to the compressor. Note that the coil geometry is the same for both the interlaced and face split evaporator, however, the tube connections or circuiting are different as shown on figure 1. Furthermore, the simulation of the injection is very CPU demanding and for this reason we chose to use only one cell per tube for the evaporator. In terms of convergence in total cooling capacity of the evaporator, this choice is within $2 \%$ of the total cooling capacity at 5 cells per tube [3]. In the condenser, refrigerant enters four channels and is mixed before entering a fifth channel. Since the circuitry is not addressed in the condenser, it is assumed to be four straight tubes. 
Table 1: Main geometry of evaporator and condenser

\begin{tabular}{lcc}
\hline & Evaporator & Condenser \\
\hline Number of coils & 2 & 1 \\
Number of channels in each coil & 2 & 5 \\
Number of tubes in each channel & 18 & 6 \\
Tube length [mm] & 444.5 & 2100 \\
Inner tube diameter [mm] & 7.6 & 7.6 \\
Outer tube diameter [mm] & 9.6 & 9.6 \\
Transverse tube pitch [mm] & 25.4 & 25 \\
Longitudinal tube pitch [mm] & 21.25 & \\
Fins & Louvred & Louvred \\
Fin pitch [mm] & 1.81 & 1.15 \\
Total outside area [m $\left.{ }^{2}\right]$ & 17.3 & 52.2 \\
Number of cells per tube & 1 & \\
\hline
\end{tabular}

Table 2: Overview of used correlations

\begin{tabular}{ll}
\hline $\begin{array}{l}\text { Air-side } \\
\text { Heat transfer }\end{array}$ & $\begin{array}{l}\text { Wang et al. (1999) } \\
\text { Fin efficiency }\end{array}$ \\
\hline Single-phase & Gnidt approximation (1949) \\
Heat transfer & Blasius (1913) \\
Friction & Ito (1960) \\
Bend friction & \\
\hline Two-phase & Shah (1982) \\
Heat transfer (evaporator) & Shah (1979) \\
Heat transfer (condenser) & Zivi (1964) \\
Void fraction & Müller-Steinhagen and Heck \\
Friction & (1986) \\
& Geary (1975) \\
Bend friction &
\end{tabular}

Each discrete cell of the evaporator is calculated as a separate heat exchanger with uniform transport properties. Mass, momentum and energy conservation equations are applied to the refrigerant in each cell, where thermodynamic equilibrium is assumed. Furthermore, changes in kinetic and potential energies are neglected. It is assumed that the tube walls have rotational symmetry (no azimuthal heat conduction) and negligible axial heat conduction. Mass and energy conservation equations are applied to the air, which is assumed to be dry. Similar assumptions are used in the condenser model of the refrigerant and airflow, however the heat resistance and the dynamics in the condenser wall are neglected. The used correlations for both the evaporator and the condenser are given in table 2. Furthermore, effectiveness-NTU relations are employed.

The expansion process is modeled as an isenthalpic process and the opening degree from equation 7 essentially controls the superheat out of the evaporator. The manifold is modeled by mixing of the refrigerant streams, i.e. mass and energy conservation equations are applied. The dynamics of the manifold wall is included and heat transfer is modeled using a constant heat transfer coefficient of $700 \mathrm{Wm}^{-2} \mathrm{~K}^{-1}$. The geometric volume flow of the compressor is $6.239 \mathrm{~m}^{3} \mathrm{~h}^{-1}$, and polynomials from the rating of the compressor are used to compute the isentropic and volumetric efficiencies.

\subsubsection{Boundary conditions}

The liquid injection model controls the overall superheat to $5 \mathrm{~K}$ by the opening degree using a PI-controller. During start-up of the simulation, the charge of the system is determined so that the subcooling becomes 2 $\mathrm{K}$. The indoor and outdoor air temperatures are $26.7^{\circ} \mathrm{C}$ and $35^{\circ} \mathrm{C}$, respectively. The mean frontal air velocities are 1.16 and $0.68 \mathrm{~m} \mathrm{~s}^{-1}$ to the evaporator and condenser, respectively.

\section{Experimental comparison}

In this section we compare the injection modeling with experiments carried out at Danfoss Nordborg. The dynamic behavior observed in the simulations showed fluctuations in important variables such as superheat and evaporating pressure. In Kærn [3] a sensitivity analysis of the fluctuations were performed in order to better understand the causes of the fluctuations, however, sensible variables such as void fraction and manifold+suction volume did not eliminate the fluctuations satisfactorily.

The fluctuations in the model have a time period corresponding to the cycle time of the liquid injection model divided by the number of channels in the evaporator (for even flow mode, see figure 4a). These fluctuations have not been observed as high in any experiments carried out at Danfoss, where the sampling frequency has been high enough to capture these fluctuations. The sampling frequency is often chosen to be $1 \mathrm{~s}^{-1}$ for refrigerant temperature and pressure measurements at Danfoss, which is too low for capturing the injection dynamics seen in the numerical model.

\subsection{The experiments}

The EcoFlow experiments were performed on a bit different system and conditions than described in previous section. The system comprised a $10.5 \mathrm{~kW}$ interlaced evaporator, a hermetic scroll compressor, microchannel condenser and an early MO disc version. The early design of the MO disc is estimated to have a flow 
Table 3: Reduced experimental boundary conditions

\begin{tabular}{ll}
\hline Superheat & $5 \mathrm{~K}$ \\
Pressure out of condenser & $31.9 \mathrm{bar}$ \\
Liquid temperature out of condenser & $45.6^{\circ} \mathrm{C}$ \\
Volume flow out of evaporator & $7.17 \mathrm{~m}^{3} \mathrm{~h}^{-1}$ \\
Indoor air temperature & $24.3^{\circ} \mathrm{C}$ \\
Indoor frontal air velocity & $2.98 \mathrm{~m} \mathrm{~s}^{-1}$ \\
\hline
\end{tabular}

ratio parameter $F_{o}$ (equation 4 ) of 0.8 , which reflects the earlier version cross-sectional areas of the main and secondary orifices. Furthermore, the flow coefficients, the step time and damping time are assumed to be the same as the final MO disc design. The cycle time was six seconds in the experiments and the flow distribution mode was even flow, see figure 4a.

These experiments are the most recent experiments carried out at Danfoss in Nordborg on a fin-andtube four channel evaporator using the EcoFlow valve. Later experiments including compensation were performed with the final EcoFlow version, however, on larger capacity units with six or eight channels each, which complicates the simulations drastically. For these reasons, the earlier EcoFlow MO experiments were chosen for the comparison. More information about the experimental data is given in Kærn [3]. The experimental data is reduced in order to be used as input to the evaporator model only, thus we only simulate the $10.5 \mathrm{~kW}$ evaporator and manifold+suction volume in this comparison. Table 3 lists the model inputs.

Figure $5 \mathrm{a}$ and $5 \mathrm{~b}$ show the experimental superheat and pressure fluctuations during three cycles. The corresponding model results are shown in figure $5 \mathrm{c}$ and $5 \mathrm{~d}$. Note that the thick curve around $5 \mathrm{~K}$ is overall superheat. Furthermore, the experiments show a bit higher individual superheats. This is because that they were measured on the tube wall surface with insulation around the tube, and may have heat entering from the surroundings.

When comparing to the experimental data, it is seen that the pressure fluctuations are smaller (approximately one third in amplitude of the numerical results). It is difficult to make this conclusion based on these experimental results, since the sample time was only $1 \mathrm{~s}^{-1}$ for the pressure. However, the experiments carried out at Danfoss with higher frequency did not show as high fluctuations as the numerical model does here. The reason for these high fluctuations in the numerical model have not been obtained so far. However, we believe that the interfacial dynamics of the twophase flow and the presence of thermodynamic nonequilibrium may be responsible for the dampening of the pressure fluctuations in the experiments. These are inherently exclusive in the mixture two-phase flow model. In addition, the refrigerant heat transfer, pressure drop and void correlations are developed from steady state experiments and employed at large transients, however, no dynamic two-phase flow correlations (pulsating flow) were found in the literature.

If we compare the individual superheat measurements and the prediction by the numerical model, then the accordance is much more acceptable. Both the measurements and the model predictions show the effect of the liquid injection into each channel, since they fluctuate similarly at a time period corresponding to the cycle time. Furthermore, the superheat decreases as the refrigerant enters through the main orifice into each channel as indicated on figure $5 \mathrm{e}$. The corresponding mass inside each channel is shown on figure 5f, which increases when the refrigerant enters through the main orifice and otherwise decreases.

What is probably most important is the individual channel overall UA-value in figure $5 \mathrm{~g}$, which shows a decrease just before new refrigerant is fed to the corresponding channel. There may be an optimization potential here if the cycle time is chosen such that the UA-value decrease is avoided. Figure 5h shows the corresponding individual channel pressure drop by friction and acceleration due to density and mass flux differences. When considering the individual channel pressure drop due to friction and acceleration, one may expect that this is the cause of the pressure fluctuations, however, the sensitivity analysis from [3] proves otherwise. It is interesting to note that the accelerational pressure drop is positive as the refrigerant is fed to each channel. This is because the refrigerant mass flow is higher at the inlet compared to the outlet of the channel, i.e. the difference in momentum flow between inlet and outlet is positive.

\section{Simulation results}

Despite the presence of the pressure fluctuations, the numerical model is used to perform simulations of the significance of the cycle time for both the multi-orifice (MO) and single-orifice (SO) designs. Furthermore, the flow ratio parameter $F_{o}$ (equation 4) for the MO design will be investigated, i.e. the flow distribution between the main and secondary orifices of the MO design. 
$\mathbf{a}$

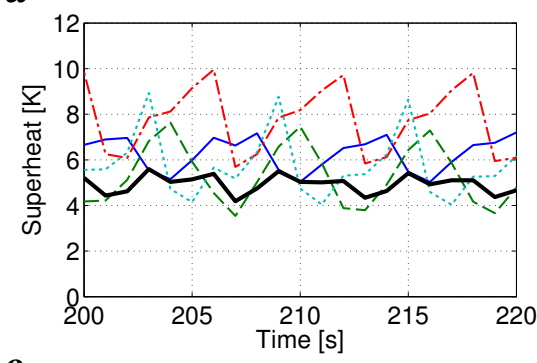

c

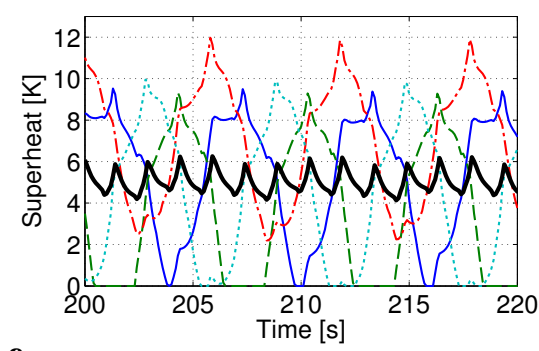

f

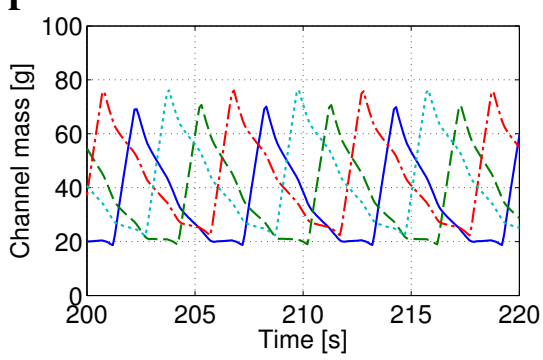

b

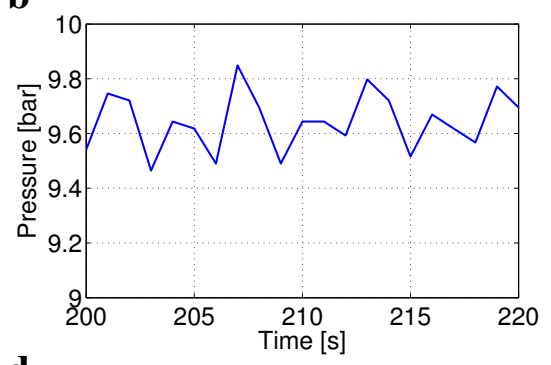

d

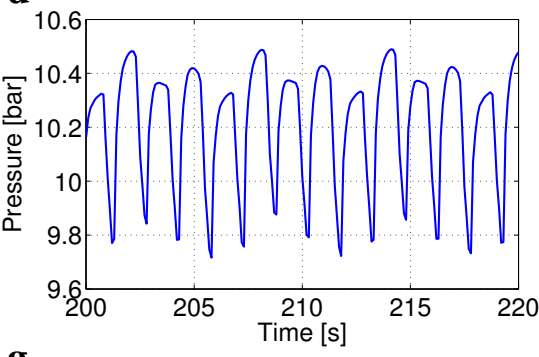

g

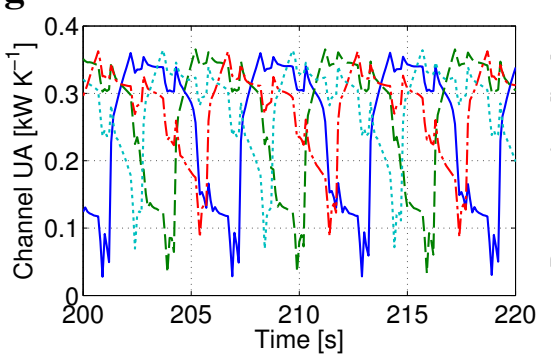

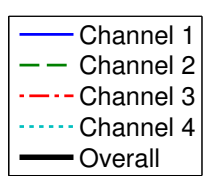

$\mathbf{e}$

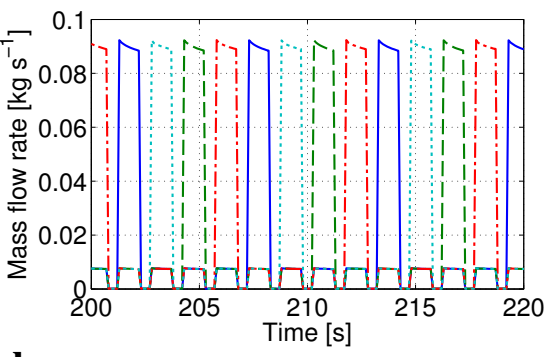

h

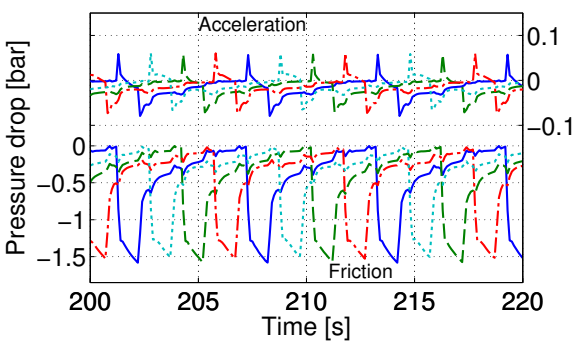

Figure 5: Zoomed-in experimental superheats and suction pressure (a,b); Model comparison (c,d) and other model results (e-h) at uniform airflow: Inlet individual channel mass flow rate (e), individual channel mass (f), individual channel overall UA-value (g) and individual channel accelerational and frictional pressure drop (h).

\subsection{Cycle time}

Figure $6(\mathrm{a}, \mathrm{b}, \mathrm{c})$ shows the UA-value, cooling capacity and $\mathrm{COP}$ using $\mathrm{MO}$ and $\mathrm{SO}$ designs as function of the cycle time. Note that the orifice flow coefficients for the 3 second cycle time simulations were assumed to be the same as for the 6 second cycle time case.

The results show that the MO design performs better than the SO design. Furthermore, the cycle time should be kept as low as possible. If flow pulsations increase heat transfer we would have expected an optimum cycle time, but it seems to be outside the cycle times considered or not shown using the current mixture model and limitations (see discussion). The simulation using the SO design at a cycle time of $20 \mathrm{sec}-$ onds failed and was not obtainable. It also seems that this case decreases the performance drastically. The question regarding which cycle time is the maximum limit is difficult to answer based on the present results. For these four channel evaporators it seems that the maximum cycle time is 10 and 6 seconds for the MO and SO design, respectively. Otherwise, the channels dry-out too much when the valve is closed.
The face split circuitry shows the best performance in contrast to the interlaced circuitry at uniform flow conditions for each distribution method. This is because the superheated regions of the face split evaporator is in the first tube row and is thus minimized. This also means that the face split evaporator performs better than the interlaced if flow maldistribution is compensated as also shown by Kærn [3].

\subsection{MO flow ratio}

Figure $6(\mathrm{~d}, \mathrm{e}, \mathrm{f})$ shows the UA-value, cooling capacity and $\mathrm{COP}$ as function of the flow ratio parameter $F_{o}$, and at a cycle time of 6 seconds. It shows that the maximum performance is when $F_{o}$ equals 0.25 , which means that the main and secondary orifices have the same dimension, thus no possibility to distribute mass individually. Essentially, all the curves on figure 4a coincides, i.e. the flow is distributed evenly to all orifices at each injection.

It shows that for uniform flow conditions, the optimal refrigerant mass flow distribution is uniform. However, the decrease in performance as $F_{o}$ increases 
$\mathbf{a}$

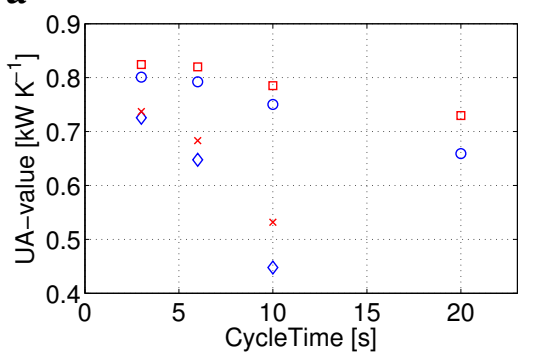

d

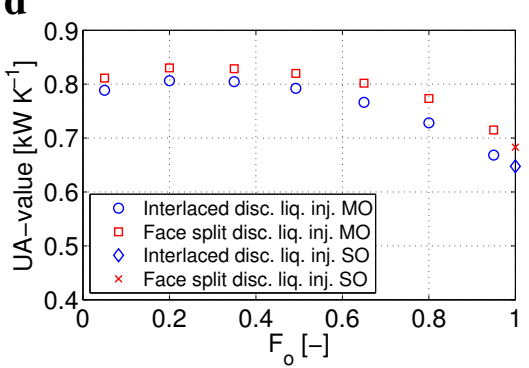

$\mathbf{b}$

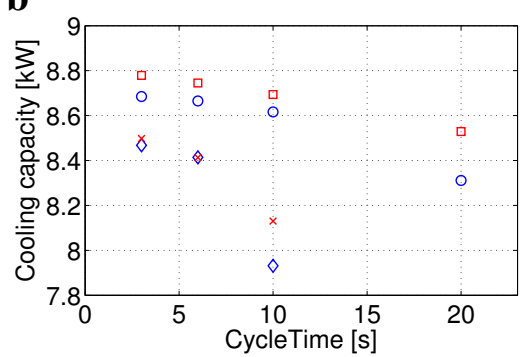

e

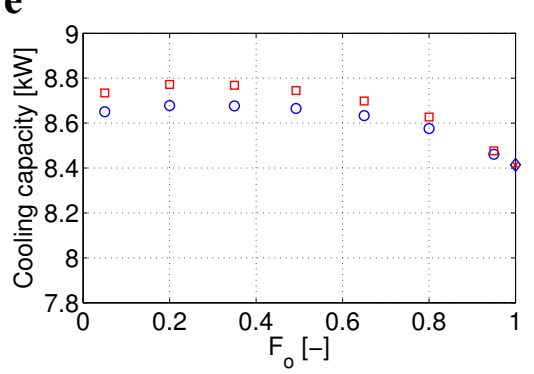

c

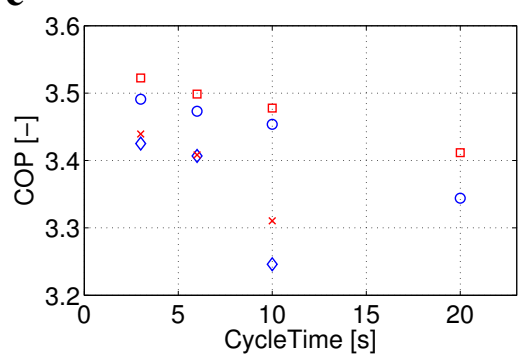

f

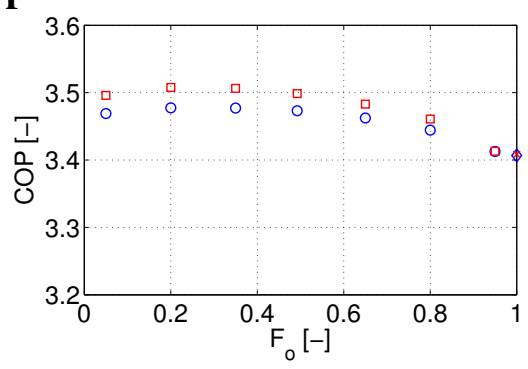

Figure 6: UA-value, cooling capacity and COP vs. the cycle time (a,b,c) at $F_{o}=0.492$ for the MO design; UA-value, cooling capacity and COP vs. the flow ratio parameter (d,e,f) at cycle time $t_{\mathrm{cyc}}=6$ seconds.

is small and the maximum limit seems to be around 0.6. Otherwise the secondary channels will also dryout too much. Furthermore, the $F_{o}=95 \%$ results of the MO design seems to be close to the SO design results presented here at $F_{o}=100 \%$.

\section{Discussion}

It is difficult to claim whether the two-phase flow pulsations increase or decrease the heat transfer mechanism. Firstly, the two-phase flow regimes are broken up by the flow pulsations and giving rise to new discontinuous flow patterns, which are not properly reflected in the steady state correlations for refrigerant heat transfer, pressure drop and void fraction. No twophase flow correlations were found in the literature by the authors that were developed for discontinuous liquid injection or pulsating flow. Secondly, the mixture two-phase flow model (also used in many Modelica libraries) showed spurious pressure fluctuations, which have not been observed as high in any experiments carried out at Danfoss. The amplitude of the fluctuations are approximately 3 times higher in the model compared to similar experiments. Thus the readers need to be cautioned that the results and conclusions from the liquid injection modeling are obtained despite the presence of these fluctuations. It is believed that the absence of the two-phase interfacial dynamics in the mixture two-phase flow model is the main cause of the high pressure fluctuations.

It needs to be stressed that it is not the finite volume model approach itself that leads to these fluctuations, but rather the governing phasic equations when added and becoming mixture equations. The model could be a separated flow model that includes the governing phasic equations and possibly the finite volume model could be used to discretize the phasic equations again. It is difficult to claim what may minimize the pressure fluctuations. The only separated flow model known to the authors that is implemented in Modelica is the work of Bauer [16], who implemented both phasic momentum equations. It resulted in another state variable (the velocity difference between the phases), which essentially is related to the void fraction. It would be interesting to look deeper into such model approaches when considering these fluctuations. Similarly, more dedicated experimental evidence of these fluctuations would be interesting to have.

\section{Conclusion}

We conclude that the typical mixture two-phase flow model that is used in many Modelica libraries is insufficient to model the discontinuous liquid injection principle (pulsating flow) into each evaporator channel. This is because the simulations showed spurious fluctuations in evaporating pressure and superheats, which have not been observed as high in any experiments carried out at Danfoss. Furthermore, it should be stressed that the correlations for heat transfer, pressure drop and void fraction employed in mixture twophase flow models do not reflect the dynamic behavior 
of the pulsating flow, since they are based upon steady state experiments. To draw detailed conclusions, further studies on the discontinuous liquid injection principle should be conducted in order to fully understand and model the phenomenon.

Despite the fluctuations, two orifice designs of the discontinuous liquid injection principle were investigated in uniform flow conditions, i.e. the multi-orifice (MO) design and the single-orifice (SO) design. The multi-orifice design allows for a secondary flow into the remaining channels at each channel injection.

The simulations of the discontinuous liquid injection principle showed that the MO design gave better performance compared to the SO design, without considering the possible individual channel defrost possibility of the SO design for the face split circuitry. In addition, the main flow and the individual secondary flows in the MO design should be kept as even as possible while having the required mass flow distribution control band. Based upon the four channel evaporator that were analyzed, it is recommended that the cycle time should be kept below 10 and 6 seconds for the MO and SO designs, respectively. Furthermore, the flow ratio parameter should be around 0.6, or adapted to specific tube circuitry according to the required mass flow distribution control band.

\section{A Refrigerant flow equations and im- plementation (evaporator model)}

This appendix describes the refrigerant flow equations and implementation for the evaporator model only. It is done in order to fully state the equations that lead to the spurious fluctuations in evaporating pressure when simulating the liquid injection principle.

\section{A.1 Mixture two-phase flow}

The model of the one-dimensional two-phase flow is the simplest form, i.e. the mixture model as derived by performing a differential analysis on each phase and adding the phasic equations [17]. The result is the mixture mass conservation, the mixture momentum conservation and the mixture energy conservation equations given by

$$
\begin{gathered}
A \frac{\partial \bar{\rho}}{\partial t}+\frac{\partial \dot{m}}{\partial z}=0 \\
\frac{\partial \dot{m}}{\partial t}+\frac{\partial}{\partial z}\left(\frac{\dot{m}^{2}}{\rho^{\prime} A}\right)=-A \frac{\partial p}{\partial z}-F_{w} A-\bar{\rho} g A \sin \theta
\end{gathered}
$$

$$
A \frac{\partial}{\partial t}(\bar{\rho} \bar{h}-p)+\frac{\partial}{\partial z}(\dot{m} h)=P q_{w}^{\prime \prime}
$$

where it has been assumed that thermodynamic equilibrium exists and that the changes in kinetic and potential energy are negligible. The mixture density, specific in situ enthalpy, specific mixed-cup enthalpy and momentum density are given by

$$
\begin{gathered}
\bar{\rho}=\rho_{g} \alpha+\rho_{f}(1-\alpha) \\
\bar{h}=\left[\rho_{f} h_{f}(1-\alpha)+\rho_{g} h_{g} \alpha\right] / \bar{\rho} \\
h=(1-x) h_{f}+x h_{g} \\
\rho^{\prime}=\left(\frac{(1-x)^{2}}{\rho_{f}(1-\alpha)}+\frac{x^{2}}{\rho_{g} \alpha}\right)^{-1}
\end{gathered}
$$

where the void fraction is defined as $\alpha=A_{g} / A$, and the vapor quality is defined as $x=\dot{m}_{g} / \dot{m}$.

Using the definition of the slip ratio, the void fraction and the vapor quality, the fundamental voidquality relation can be derived as

$$
\begin{aligned}
S & =\frac{U_{g}}{U_{f}}=\frac{\frac{\dot{m}_{g}}{\rho_{g} \alpha A}}{\frac{\dot{m}_{f}}{\rho_{f}(1-\alpha) A}} \\
& =\frac{x}{1-x} \frac{\rho_{f}}{\rho_{g}} \frac{1-\alpha}{\alpha}
\end{aligned}
$$

and rewritten in terms of the void fraction as

$$
\alpha=\left[1+\frac{\rho_{g}}{\rho_{f}} \frac{1-x}{x} S\right]^{-1}
$$

If homogeneous flow is assumed, then $S=1$ and the homogeneous void fraction, $\alpha_{H}$, may be calculated by equation 16. Furthermore, for homogeneous flow it can be shown that $\bar{h}=h$ and $\rho^{\prime}=\bar{\rho}=\rho_{H}$ by using the homogeneous void fraction, where the homogeneous mixture density, $\rho_{H}$, becomes

$$
\rho_{H}=\left(\frac{x}{\rho_{g}}+\frac{1-x}{\rho_{f}}\right)^{-1}
$$

The state variables are chosen to be $\bar{h}$ and $p$. The derivative of the mixture density with respect to time is computed by use of the chain rule

$$
\frac{\partial \bar{\rho}}{\partial t}=\left.\frac{\partial \bar{\rho}}{\partial p}\right|_{\bar{h}} \frac{\partial p}{\partial t}+\left.\frac{\partial \bar{\rho}}{\partial \bar{h}}\right|_{p} \frac{\partial \bar{h}}{\partial t}
$$

where the partial derivatives of mixture density with respect to pressure and in situ enthalpy are calculated by numerical finite difference as 


$$
\begin{aligned}
& \left.\frac{\partial \bar{\rho}}{\partial p}\right|_{\bar{h}}=\frac{\bar{\rho}(p+\Delta p, \bar{h})-\bar{\rho}(p, \bar{h})}{\Delta p} \\
& \left.\frac{\partial \bar{\rho}}{\partial \bar{h}}\right|_{p}=\frac{\bar{\rho}(p, \bar{h}+\Delta \bar{h})-\bar{\rho}(p, \bar{h})}{\Delta \bar{h}}
\end{aligned}
$$

Equations 8, 9 and 10 are discretized according to the Finite Volume Method (FVM), where the number of control volumes must be high enough to resolve the spatial distribution of properties.

The staggered grid structure is adopted as described by Patankar [18]. It means that the mass and energy conservation will be solved on the control volume grid, and the momentum equation will be solved on a staggered grid as depicted on figure 7 , where $\psi$ denotes a thermodynamic quantity and $\hat{\psi}$ its approximation. Similar discretization methodology was used in Bauer [16].

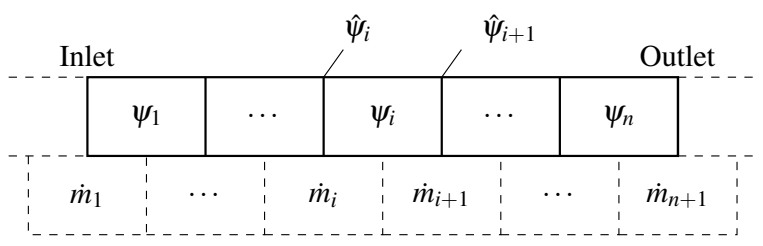

Figure 7: Staggered grid structure; thick $=$ control volume grid, dashed $=$ staggered grid

The mass and energy conservation equations become

$$
\begin{gathered}
A \Delta z \frac{d \bar{\rho}_{i}}{d t}=\dot{m}_{i}-\dot{m}_{i+1} \\
A \Delta z \frac{d}{d t}\left(\bar{\rho}_{i} \bar{h}_{i}-p_{i}\right)=\dot{H}_{i}-\dot{H}_{i+1}+\dot{Q}_{i}
\end{gathered}
$$

where the enthalpy flow $\dot{H}_{i}=\dot{m}_{i} \hat{h}_{i}$ and heat flow $\dot{Q}_{i}=$ $P \Delta z q_{w, i}^{\prime \prime}=P \Delta z h_{\mathrm{tc}, \mathrm{i}}\left(T_{w, i}-T_{i}\right)$ have been used, and Newton's law of cooling is applied with the well known heat transfer coefficient $h_{\mathrm{tc}}$.

For convection dominated flows the upwind difference scheme is recommended to approximate thermodynamic quantities onto the staggered grid, because central difference scheme may lead to non-physical solutions. The $1^{\text {st }}$ order upwind scheme is obtained by taking the control volume face value (staggered grid center) to be equal to the nearest upstream control volume center, thus

$$
\hat{\psi}_{i} \approx \delta_{i} \psi_{i}+\left(1-\delta_{i}\right) \psi_{i-1} \quad i=1 . . n+1
$$

where $\delta_{i}$ is the indicator function denoting the direction of the mass flow

$$
\delta_{i}=\left\{\begin{array}{cc}
0 & \dot{m} \geq 0 \\
1 & \dot{m}<0
\end{array}\right.
$$

The momentum equation becomes

$$
\begin{array}{r}
\Delta z \frac{d \dot{m}_{i}}{d t}=\Delta \dot{I}_{i}-A\left(p_{i}-p_{i-1}\right)-F_{w, i} A \Delta z \\
-\hat{\bar{\rho}}_{i} g A \Delta z \sin \theta
\end{array}
$$

where the momentum flow $\dot{I}_{i}=\dot{m}_{i}^{2} /\left(\hat{\rho}_{i}^{\prime} A\right)$ has been used and the difference in momentum flow, $\Delta \dot{I}_{i}$, is approximated according to the $2^{\text {nd }}$ order central difference scheme as

$$
\Delta \dot{I_{i}} \approx \frac{\left(\dot{I}_{i-1}-\dot{I}_{i}\right)+\left(\dot{I}_{i}-\dot{I}_{i+1}\right)}{2}=\frac{d \dot{I}_{i-1}+d \dot{I}_{i}}{2}
$$

where $d \dot{I}$ is the momentum flow difference between the staggered grid cells. The use of the central difference scheme serves to avoid discontinuities in the momentum equation.

Boundary models are used to compute the boundary conditions $\dot{H}, \dot{I}, d \dot{I}, \hat{\psi}$. The change of momentum flow $d \dot{I}$ at the inlet or outlet is simply set to zero, whereas the other variables are computed from the thermodynamic state and the mass flow rate.

Correlations for the frictional force, $F_{w}$, the heat transfer coefficient, $h_{\mathrm{tc}}$, and the void fraction, $\alpha$, must be supplied to close the system of equations.

\section{A.2 Tube wall}

The tube wall is discretized according to the Resistance Capacitance Method [19]. The method essentially uses the thermal resistances to describe the heat flows across the tube wall boundaries. The tube wall is assumed to have rotational symmetry, i.e. $T=T(r, z)$, and thus the energy equation for each discrete cell becomes

$$
M c_{p} \frac{d T}{d t}=\dot{Q}_{W}+\dot{Q}_{E}+\dot{Q}_{S}+\dot{Q}_{N}
$$

where $\dot{Q}_{S}=-P \Delta z q_{w}^{\prime \prime}$ from equation 10 . The entering and leaving heat flows are depicted on figure 8 .

By definition, the heat flows are computed as $\dot{Q}=$ $\Delta T / R$, where the thermal resistances in the radial and axial directions to the midpoint of the wall cell are

$$
\begin{gathered}
R_{\mathrm{ax}}=0.5 \frac{\Delta z}{k A} \\
R_{\mathrm{rad}}=0.5 \frac{\ln \frac{D / 2}{d / 2}}{2 \pi k \Delta z}
\end{gathered}
$$



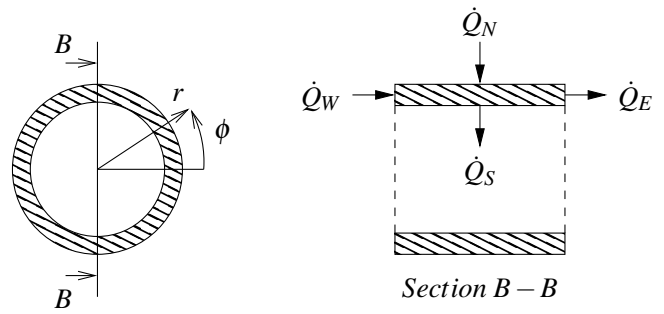

Figure 8: Heat flows to and from the tube wall

The boundary condition at the inlet and outlet of the pipe wall is simply no heat flow in the axial direction. Since we only use one cell per tube in this study the axial heat conduction is essentially neglected.

\section{A.3 Airflow}

The airflow is assumed to be incompressible and can not accumulate mass or energy. With these assumptions, the mass and energy conservation equation for each air cell become

$$
\begin{gathered}
\dot{m}_{\text {in }}-\dot{m}_{\text {out }}=0 \\
\left(\dot{m} c_{p} T\right)_{\text {in }}-\left(\dot{m} c_{p} T\right)_{\text {out }}+\dot{Q}_{N}=0
\end{gathered}
$$

The effectiveness-NTU method is applied to describe the variation in air temperature, i.e. the single stream heat exchanger configuration where the surface temperature of each cell is uniform. It describes the actual heat flow by the effectiveness, $\varepsilon$, of the highest possible heat transfer, i.e.

$$
\dot{Q}_{N}=\varepsilon C_{\min }\left(-\Delta T_{\max }\right)
$$

where $C_{\min }$ is the minimum capacitance flow and $\Delta T_{\max }$ is the maximum temperature difference. Correlations for the heat transfer coefficient and the fin efficiency must be applied to compute the Number of Transfer Units and thus the effectiveness.

\section{A.4 Smooth functions}

A first order continuous function is applied at the phase transitions $(0 \leq x<0.05$ and $0.95<x \leq 1)$. The function ensures a smooth transition from two-phase to single phase in heat transfer and frictional pressure drop correlations. If the transitions are discontinuous, the equation solver might be slow or even fail to converge. The first order continuous function is described in Richter [10]. The used correlations are shown in table 2.

\section{A.5 Heat exchanger architecture}

Components of the refrigerant (both control volume grid cell and staggered grid cell), the wall and the air have been made in Dymola, and essentially arrays of these components are put together to form the evaporator in cross flow operation, as shown on figure 9.

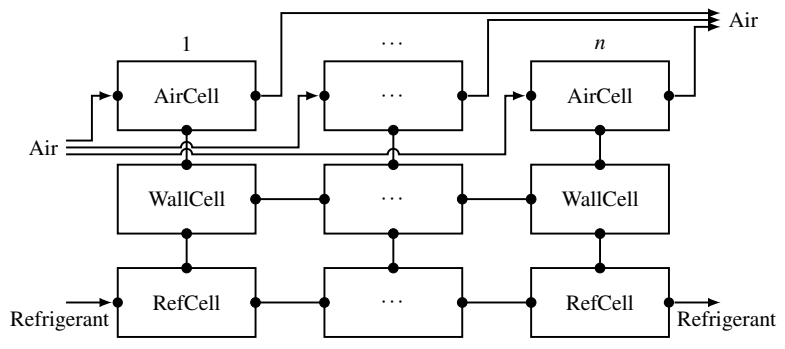

Figure 9: Heat exchanger architecture; cross flow.

Following this implementation, we did not use already made components from the Modelica standard library. We chose this to learn every step of the implementation in Modelica and to be able to quickly apply changes to the model formulation and correlations if necessary. Furthermore, we did not use the Modelica stream prefix. Since the compressor runs at constant speed, we did not observe flow reversal during the flow pulsations.

The circuitry modeling is a bit more complex than shown on figure 9 , however, its construction is simply a matter of running through many for loops to connect the airflow paths and the refrigerant bends (assumed adiabatic) with correct radius. Note that the refrigerant flow is discretized fully from inlet to outlet through the bends such that the bends also contains a volume grid cell and a staggered grid cell. More information on the circuitry implementation is given in Kærn [3].

\section{References}

[1] W. V. Payne, P. A. Domanski, Potential benefits of smart refrigerant distributors, Final report No. ARTI-21CR/610-20050-01, AirConditioning and Refrigeration Technology Institute, Arlington, VA, USA (2003).

[2] J.-H. Kim, J. E. Braun, E. A. Groll, Evaluation of a hybrid method for refrigerant flow balancing in multi-circuit evaporators, International Journal of Refrigeration 32 (2009) 1283 - 1292.

[3] M. R. Kærn, Analysis of flow maldistribution in fin-and-tube evaporators for residential airconditioning systems, Ph.D. thesis, Technical 
University of Denmark, Department of Mechanical Engineering, Kgs. Lyngby, Denmark (2011).

[4] P. A. Domanski, D. Yashar, Application of an evolution program for refrigerant circuitry optimization, in: ACRECONF "Challenges To Sustainability", New Delhi, India, 2007.

[5] T. Funder-Kristensen, H. Nicolaisen, J. Holst, M. H. Rasmussen, J. H. Nissen, Refrigeration system, US Patent, Pub. No.: US 2009/0217687 A1 (2009).

[6] G. Mader, C. Thybo, An electronic expansion valve with automatic refrigerant distribution control, in: Deutsche Kälte-Klima-Tagung, Magdeburg, Germany, 2010.

[7] R. W. Fox, A. T. McDonald, P. J. Pritchard, Introduction to fluid mechanics, Wiley, New York, 2004.

[8] C. Park, H. Cho, Y. Lee, Y. Kim, Mass flow characteristics and empirical modeling of R22 and R410A flowing through electronic expansion valves, International Journal of Refrigeration 30 (8) (2007) 1401-1407.

[9] L. Chen, J. Liu, J. Chen, Z. Chen, A new model of mass flow characteristics in electronic expansion valves considering metastability, International Journal of Thermal Sciences 48 (6) (2009) $1235-1242$.

[10] C. C. Richter, Proposal of new object-oriented equation-based model libraries for thermodynamic systems, Ph.D. thesis, Technische Universität Carolo-Wilhelmina zu Braunschweig, Fakultät für Maschinenbau (2008).

[11] M. R. Kærn, B. Elmegaard, L. F. S. Larsen, Experimental comparison of the dynamic evaporator response using homogeneous and slip flow modelling, in: 8th International Modelica Conference, Dresden, Germany, 2011.

[12] Dynasim AB, Research Park Ideon SE-223 70, Lund, Sweden, Dynamic Modeling Laboratory, Dymola User's Manual, version 7.4 (2010).

[13] J. Eborn, H. Tummescheit, K. Prölss, Airconditioning - a modelica library for dynamic simulation of ac systems, in: 4th International Modelica Conference, Hamburg, Germany, 2005, pp. 185 192.
[14] M. J. Skovrup, Thermodynamic and thermophysical properties of refrigerants, Department of Energy Engineering, Technical University of Denmark, Nils Koppels Allé, Building 402, DK-2800 Lyngby, Denmark (2009).

[15] W.-J. Zhang, C.-L. Zhang, A generalized moving-boundary model for transient simulation of dry-expansion evaporators under larger disturbances, International Journal of Refrigeration 29 (2006) 1119 - 1127.

[16] O. Bauer, Modelling of two-phase flows with modelica, Master's thesis, Lund University, Department of Automatic Control (1999).

[17] S. M. Ghiaasiaan, Two-phase flow: Boiling and Condensation in Conventional and Miniature Systems, 1st Edition, Cambridge University Press, 2008.

[18] S. V. Patankar, Numerical heat transfer and fluid flow, Taylor \& Francis, 1980.

[19] A. F. Mills, Heat Transfer, 2nd Edition, Prentice Hall, 1999. 